\title{
Research Article \\ Fixed Points for Pseudocontractive Mappings on Unbounded Domains
}

\author{
Jesús García-Falset and E. Llorens-Fuster \\ Departamento de Análisis Matemático, Facultad de Matemáticas, Universitat de Valencia, \\ 46100 Burjassot, Spain \\ Correspondence should be addressed to Jesús García-Falset, garciaf@uv.es \\ Received 4 September 2009; Accepted 14 October 2009 \\ Academic Editor: Mohamed A. Khamsi
}

Copyright (C 2010 J. García-Falset and E. Llorens-Fuster. This is an open access article distributed under the Creative Commons Attribution License, which permits unrestricted use, distribution, and reproduction in any medium, provided the original work is properly cited.

We give some fixed point results for pseudocontractive mappings on nonbounded domains which allow us to obtain generalizations of recent fixed point theorems of Penot, Isac, and Németh. An application to integral equations is given.

\section{Introduction}

Let $C$ be a nonempty subset of a Banach space $X$ with norm $\|\cdot\|$. Recall that a mapping $T: C \rightarrow X$ is said to be nonexpansive whenever $\|T(x)-T(y)\| \leq\|x-y\|$ for every $x, y \in C$. $X$ is said to have the fixed point property ((FPP) for short) if every nonexpansive selfmapping of each nonempty bounded closed and convex subset of $X$ has a fixed point. It has been known from the outset of the study of this property (around the early sixties of the last century) that it depends strongly on "nice" geometrical properties of the space. For instance, a celebrated result due to Kirk [1] establishes that those reflexive Banach spaces with normal structure (NS) have the (FPP). In particular, uniformly convex Banach spaces have normal structure (see $[2,3]$ for more information).

If $C$ is a closed convex of a Banach space enjoying the (FPP), in general it is not true that $T: C \rightarrow C$ has a fixed point due to the possible unboundedness of $C$ (it is enough to consider any translation map, with nonnull vector, in the Banach space X). In 2003 Penot [4] showed that if $C$ is a closed convex subset of a uniformly convex Banach space $X, T: C \rightarrow C$ is a nonexpansive mapping, and for some $x_{0} \in C$,

$$
\limsup _{x \in C,\|x\| \rightarrow \infty} \frac{\left\|T(x)-T\left(x_{0}\right)\right\|}{\left\|x-x_{0}\right\|}<1
$$

(in other words if $T$ is asymptotically contractive), then $T$ has a fixed point. 
A celebrated fixed point result due to Altman [5] is the following.

Let $H$ be a separable Hilbert space, with inner product $[\cdot, \cdot]$ and induced norm $\|x\|=$ $\sqrt{[x, x]}$. Let $F: B_{r} \rightarrow H$ be a weakly closed mapping where $B_{r}$ is the closed ball with center 0 and radius $r$. Suppose that $T$ maps the sphere $S_{r}$ into a bounded set in $H$. If the following condition is satisfied:

$$
[T(x), x] \leq[x, x]
$$

for all $x \in S_{r}$, then $T$ has a fixed point in $B_{r}$.

In 2006, Isac and Németh [6] gave some fixed point results for nonexpansive nonlinear mappings in Banach spaces inspired by Penot's results where the asymptotically contractiveness was stated in similar terms to condition (1.2).

In this paper we generalize some Penot, Isac, and Németh's fixed point results in several ways. First, we will be concerned with pseudocontractive mappings, a more general class of mappings than the nonexpansive ones. Second, we use an inwardness condition weaker than $T(C) \subset C$, and finally our Altmann type assumptions are more general than those required in $[4,6]$.

We prove our fixed point results as a consequence of some results on the existence of zeroes for accretive operators. Among the problems treated by accretive operators theory, one of the most studied is just this one (see, e.g., Kirk and Schöneberg's paper [7] as well as $[3,8,9]$ and the references therein). We obtain here several results of this type, and in particular we give a characterization in the setting of the Banach spaces with (FPP) of those $m$-accretive operators which have zeroes.

\section{Preliminaries}

Throughout this paper we suppose that $X$ is a real Banach space and that $X^{*}$ is its topological dual. We use $B_{r}$ to denote the closed ball centered at $0_{X} \in X$ with radius $r>0$. We also use the notation $|B|:=\inf \{\|y\|: y \in B\}, B \subset X$.

If $x \in X$, we will denote by $J(x)$ the normalized duality mapping at $x$ defined by $J(x):=\left\{j \in X^{*}: j(x)=\|x\|^{2},\|j\|=\|x\|\right\}$. We will often use the mapping $\langle\cdot, \cdot\rangle_{+}: X \times X \rightarrow \mathbb{R}$ defined by $\langle y, x\rangle_{+}:=\max \{j(y): j \in J(x)\}$.

A mapping $A: D(A) \rightarrow 2^{X}$ will be called an operator on $X$. The domain of $A$ is denoted by $D(A)$ and its range by $R(A)$. It is well known that an operator $A: D(A) \subset X \rightarrow$ $2^{X}$ is accretive if and only if $\langle u-v, x-y\rangle_{+} \geq 0$ for all $(x, u),(y, v) \in A$.

If, in addition, $R(I+\lambda A)$ is for one, hence for all, $\lambda>0$, precisely $X$, then $A$ is called $m$-accretive. We say that $A$ satisfies the range condition if $\overline{D(A)} \subset R(I+\lambda A)$ for all $\lambda>0$.

We now recall some important facts regarding accretive operators which will be used in our paper (see, e.g., [10]).

Proposition 2.1. Let $A: D(A) \rightarrow 2^{X}$ be an operator on $X$. The following conditions are equivalent:

(i) $A$ is an accretive operator,

(ii) the inequality $\|x-y\| \leq|x-y+\lambda(A(x)-A(y))|$ holds for all $\lambda \geq 0$, and for every $x, y \in D(A)$,

(iii) for each $\lambda>0$ the resolvent $J_{\lambda}:=(I+\lambda A)^{-1}: R(I+\lambda A) \rightarrow D(A)$ is a single-valued nonexpansive mapping. 
Let $C$ be a nonempty subset of $X$ and let $T: C \rightarrow X$ be a mapping. Recall that a sequence $\left(x_{n}\right)$ of elements of $C$ is said to be an a.f.p sequence for $T$ whenever $\lim _{n \rightarrow \infty} \| x_{n}-$ $T\left(x_{n}\right) \|=0$. It is well known that if $T$ is a nonexpansive mapping which maps a closed convex bounded subset $C$ of $X$ into itself, then such a mapping always has a.f.p. sequences in $C$.

When the Banach space $X$ has the (FPP), Morales [9] gave a characterization of those $m$-accretive operators $A$ such that $0_{X} \in R(A)$. Let us recall such result.

Theorem 2.2. Let $X$ be a Banach space with the FPP, and let $A: D(A) \rightarrow 2^{X}$ be an $m$-accretive operator. Then $0_{X} \in R(A)$ if and only if the set $E=\{x \in D(A): t x \in A(x), t<0\}$ is bounded.

A mapping $T: C \rightarrow X$ is said to be pseudocontractive if for every $x, y \in C$, and for all positive $r,\|x-y\| \leq\|(1+r)(x-y)-r(T(x)-T(y))\|$. Pseudocontractive mappings are easily seen to be more general than nonexpansive mappings ones. The interest in these mappings also stems from the fact that they are firmly connected to the well-known class of accretive mappings. Specifically $T$ is pseudocontractive if and only if $I-T$ is accretive where $I$ is the identity mapping.

We say that a mapping $T: C \rightarrow X$ is demiclosed at $0_{X}$ if for any sequence $\left(x_{n}\right)$ in $C$ weakly convergent to $x_{0} \in C$ with $\left(T\left(x_{n}\right)\right)$ norm convergent to $0_{\mathrm{X}}$ one has that $T\left(x_{0}\right)=0_{\mathrm{X}}$. It is well known that if $C$ is weakly compact and convex, $T: C \rightarrow C$ is nonexpansive, and $I-T: C \rightarrow X$ is demiclosed at $0_{X}$, then $T$ has a fixed point in $C$.

We say that the mapping $T: C \rightarrow X$ is weakly inward on $C$ if $\lim _{\lambda \rightarrow 0^{+}} d((1-\lambda) x+$ $\lambda T(x), C)=0$ for all $x \in C$. Such condition is always weaker than the assumption of $T$ mapping the boundary of $C$ into $C$. Recall that if $A: D(A) \rightarrow X$ is a continuous accretive mapping, $D(A)$ is convex and closed, and $I-A$ is weakly inward on $D(A)$, then $A$ has the range condition (see [11]).

We say that a semi-inner-product is defined on $X$, if to any $x, y \in X$ there corresponds a real number denoted by $[x, y]$ satisfying the following properties:

(s1) $[x+y, z]=[x, z]+[y, z]$ for $x, y, z \in X$,

(s2) $[\lambda x, y]=\lambda[x, y]$ for $x, y \in X$, and $\lambda \in \mathbb{R}$,

(s3) $[x, x]>0$ for $x \neq 0$,

(s4) $|[x, y]|^{2} \leq[x, x][y, y]$.

It is known (see $[12,13])$ that a semi-inner-product space is a normed linear space with the norm $\|x\|_{s}=[x, x]^{1 / 2}$ and that every Banach space can be endowed with a semi-innerproduct (and in general in infinitely many different ways, but a Hilbert space in a unique way).

In [6] the authors considered several fixed point results for nonexpansive mappings with unbounded domains satisfying additional asymptotic contractive-type conditions in terms of a function $G: X \times X \rightarrow \mathbb{R}$ under the following assumptions:

(G1) $G(\lambda x, y)=\lambda G(x, y)$ for any $x, y \in X$ and $\lambda>0$,

(G2) $\|x\|^{2} \leq G(x, x)$ for any $x \in X$,

(G3) $G(x+y, z)=G(x, z)+G(y, z)$ for any $x, y, z \in X$,

(G4) there exists an $M>0$ such that $|G(x, y)| \leq M\|x\|\|y\|$ for every $x, y \in X$. 


\section{Zeroes for Accretive Operators}

We begin with the definition of a certain kind of functions on which we will be concerned. This class is more general than the corresponding one considered in [6]. Let $(X,\|\cdot\|)$ be a real Banach space and $G: X \times X \rightarrow \mathbb{R}$ a mapping which satisfies the following conditions:

(g1) $G(\lambda x, y) \leq \lambda G(x, y)$ for any $x, y \in X$ and $\lambda>0$,

(g2) there exists $S>0$ such that $0<G(x, x)$ for any $x \in X$ with $\|x\| \geq S$,

(g3) $G(x+y, z) \leq G(x, z)+G(y, z)$ for any $x, y, z \in X$,

(g4) for each $y \in X$, there exists $t>0$ (depending on $y$ ), such that if $\|x\| \geq t$, then $|G(y, x)|<G(x, x)$. (g1)-(g4).

Notice that if we consider either $G(x, y)=[x, y]$ or $G(x, y)=\langle x, y\rangle_{+}$, then $G$ satisfies

Let $X$ be a Banach space with the (FPP). If $A: D(A) \rightarrow 2^{X}$ is an $m$-accretive operator such that its domain $D(A)$ is a bounded set, then it is well known that $0_{\mathrm{X}} \in R(A)$ (see, e.g., $[7,9])$. If $D(A)$ is not bounded, then we give the following result.

Theorem 3.1. Let $X$ be a Banach space with the (FPP). Let $G: X \times X \rightarrow \mathbb{R}$ be a mapping satisfying (g1) and (g2). If $A: D(A) \rightarrow 2^{X}$ is an m-accretive operator such that there exists $R>0$ with

$$
\sup _{y \in A x} G(x-y, x) \leq G(x, x)
$$

whenever $\|x\| \geq R$, then, $0_{\mathrm{X}} \in R(A)$.

Proof. Since $A$ is $m$-accretive and $X$ has the (FPP), by Theorem 2.2 we know that $0_{X} \in R(A)$ if and only if the set $E=\{x \in D(A): t x \in A(x) ; t<0\}$ is bounded.

In order to get a contradiction we assume that $E$ is an unbounded set. This fact means that for each $n \in \mathbb{N}$ there exists $x_{n} \in E$ such that $\left\|x_{n}\right\| \geq n$.

Since $x_{n} \in E$, then there exist $t_{n}<0$ and $y_{n} \in A\left(x_{n}\right)$ such that $t_{n} x_{n}=y_{n}$. This means that $\left(1-t_{n}\right) x_{n}=x_{n}-y_{n}$.

Consequently, for every $n \geq \max \{R, S\}$, we have

$$
0<G\left(x_{n}, x_{n}\right)=G\left(\frac{1}{1-t_{n}}\left(x_{n}-y_{n}\right), x_{n}\right) \leq \frac{1}{1-t_{n}} G\left(x_{n}-y_{n}, x_{n}\right) \leq \frac{1}{1-t_{n}} G\left(x_{n}, x_{n}\right),
$$

which is a contradiction.

In the following theorem we are going to give a characterization in terms of a particular function $G$, (in the framework of the Banach spaces with the (FPP)), of those $m$-accretive operators which have zeroes.

Theorem 3.2. Let $X$ be a Banach space with the (FPP). Let $G: X \times X \rightarrow \mathbb{R}$ be the mapping

$$
G(x, y)= \begin{cases}\lambda, & \text { if } x=\lambda y, \lambda>0, x \neq 0, \\ 0, & \text { otherwise. }\end{cases}
$$


If $A: D(A) \rightarrow 2^{\mathrm{X}}$ is an m-accretive operator, then the following conditions are equivalent:

(1) there exists $R>0$ such that $\sup _{y \in A x} G(x-y, x) \leq G(x, x)$ whenever $x \in D(A)$ and $\|x\| \geq R$

(2) $0_{X} \in R(A)$.

Proof. (1) $\Rightarrow(2)$ It is clear that $G$ satisfies conditions (g1) and (g2), thus by Theorem 3.1 we obtain that $0 \in R(A)$.

$(2) \Rightarrow(1)$ In order to get a contradiction, assume that for each $n \in \mathbb{N}$ there exists $x_{n} \in$ $D(A)$ with $\left\|x_{n}\right\| \geq n$ such that

$$
\sup _{y_{n} \in A\left(x_{n}\right)} G\left(x_{n}-y_{n}, x_{n}\right)>G\left(x_{n}, x_{n}\right)=1
$$

The above inequality implies that for each $n \in \mathbb{N}$, there exist $y_{n} \in A\left(x_{n}\right)$ and $\lambda_{n}>1$ such that $G\left(x_{n}-y_{n}, x_{n}\right)=\lambda_{n}$.

By definition of $G$, we have that $x_{n}-y_{n}=\lambda_{n} x_{n}$, and thus $\left(1-\lambda_{n}\right) x_{n}=y_{n} \in A\left(x_{n}\right)$.

From the above fact, we derive that for each $n \in \mathbb{N}$,

$$
x_{n} \in E=\{x \in D(A): t x \in A(x), t<0\},
$$

that is, $E$ is unbounded. By Theorem 2.2, it follows that if $E$ is unbounded, then $0_{X} \notin R(A)$; therefore, we have a contradiction.

As a consequence of the above characterization it is easy to capture the following result which is related to [7, Theorems 2 and 3].

Corollary 3.3. Let $X$ be a real Banach space with the (FPP). Suppose that $A: D(A) \subseteq X \rightarrow 2^{X}$ is an $m$-accretive operator for which there exist $x_{0} \in D(A)$ and $R>0$ such that

$$
\left|A\left(x_{0}\right)\right|<|A(x)|
$$

for all $x \in D(A)$ with $\|x\| \geq R$. Then $0_{X} \in R(A)$.

Proof. Without loss of generality we may assume that $x_{0}=0_{X}$. Otherwise, we work with the operator $\tilde{A}: D(A) \backslash\left\{x_{0}\right\} \rightarrow 2^{X}$ defined by $\tilde{A}\left(x-x_{0}\right)=A(x)$.

If we take $G(\cdot, \cdot)$ as in Theorem 3.2, to obtain the conclusion it is enough to see that

$$
\sup _{y \in A(x)} G(x-y, x) \leq G(x, x)=1
$$

whenever $x \in D(A) \backslash B_{R}$.

In order to get a contradiction, assume that for each $n \in \mathbb{N}$ there exists $x_{n} \in D(A)$ with $\left\|x_{n}\right\| \geq n$ such that

$$
\sup _{y_{n} \in A\left(x_{n}\right)} G\left(x_{n}-y_{n}, x_{n}\right)>G\left(x_{n}, x_{n}\right)=1
$$


The above inequality implies that for each $n \in \mathbb{N}$, there exist $y_{n} \in A\left(x_{n}\right)$ and $\lambda_{n}>1$ such that $G\left(x_{n}-y_{n}, x_{n}\right)=\lambda_{n}$.

By definition of $G$, we have that $x_{n}-y_{n}=\lambda_{n} x_{n}$, and thus $\left(1-\lambda_{n}\right) x_{n}=y_{n} \in A\left(x_{n}\right)$.

By hypothesis, we know that the inequality $\left|A\left(0_{X}\right)\right|<\left\|y_{n}\right\|=\left(\lambda_{n}-1\right)\left\|x_{n}\right\|$ holds for every $n \geq R$.

This means that there exists $z_{n} \in A\left(0_{X}\right)$ such that $\left\|z_{n}\right\|<\left\|y_{n}\right\|$. Therefore

$$
\left(1-\lambda_{n}\right)\left\|x_{n}\right\|<-\left\|z_{n}\right\|
$$

On the other hand, since $A$ is an accretive operator, it is clear that

$$
\begin{aligned}
0 \leq\left\langle y_{n}-z_{n}, x_{n}-0_{X}\right\rangle_{+} & =\left\langle\left(1-\lambda_{n}\right) x_{n}-z_{n}, x_{n}\right\rangle_{+} \\
& \leq\left(1-\lambda_{n}\right)\left\|x_{n}\right\|^{2}+\left\|z_{n}\right\|\left\|x_{n}\right\| \\
& \leq\left(\left(1-\lambda_{n}\right)\left\|x_{n}\right\|+\left\|z_{n}\right\|\right)\left\|x_{n}\right\|<0,
\end{aligned}
$$

which is a contradiction.

The above corollary allows us to recapture the following well-known result.

Corollary 3.4. Let $X$ be a real Banach space with the (FPP). Suppose that $A: D(A) \subseteq X \rightarrow 2^{X}$ is an m-accretive operator; if

$$
\lim _{\substack{\|x\| \rightarrow \infty \\ x \in D(A)}}|A(x)|=\infty
$$

then $0_{\mathrm{X}} \in R(A)$.

Corollary 3.5. Let $X$ be a Banach space with the (FPP). Let $G: X \times X \rightarrow \mathbb{R}$ be a mapping satisfying (g1) and (g2). If $A: D(A) \rightarrow 2^{X}$ is an $m$-accretive operator such that

$$
\lim _{\substack{x \in D(A),\|x\| \rightarrow \infty}} \sup _{y \in A x} \frac{G(x-y, x)}{G(x, x)}<1
$$

then $0_{\mathrm{X}} \in R(A)$.

Proof. It is clear that condition (3.12) implies assumption (3.1).

Corollary 3.6. Let $H$ be a real Hilbert space. Let $\varphi: H \rightarrow(-\infty, \infty]$ be a convex proper lower semicontinuous mapping with effective domain $D(\varphi)$. Suppose that for some $z_{0} \in D(\varphi)$ there exists $r>0$ such that $\varphi\left(z_{0}\right)<\varphi(x)$ for all $x \in H$ with $\|x\| \geq r$. Then $\varphi$ has an absolute minimum on $H$.

Proof. Consider $\partial \varphi: H \rightarrow 2^{H}$ the subdifferential associated to $\varphi$, that is

$$
\partial \varphi(x)=\{y \in H:[y, z-x] \leq \varphi(z)-\varphi(x), \forall z \in H\} .
$$


It is well known that $\partial \varphi$ is an $m$-accretive operator on $H$ (see [14]). Now, we consider $G$ : $H \times H \rightarrow \mathbb{R}$ defined as in Theorem 3.2.

In order to get a contradiction, suppose that given $n \in \mathbb{N}$ there is $x_{n} \in D(\partial \varphi)$ with $\left\|x_{n}\right\| \geq n$ such that

$$
\sup _{y_{n} \in \partial \varphi\left(x_{n}\right)} G\left(x_{n}-y_{n}, x_{n}\right)>G\left(x_{n}, x_{n}\right)
$$

By definition of $G$ we have that there exists $y_{n} \in \partial \varphi\left(x_{n}\right)$ such that

$$
G\left(x_{n}-y_{n}, x_{n}\right)=\lambda_{n}>1
$$

This means that $x_{n}-y_{n}=\lambda_{n} x_{n}$, hence $\left(1-\lambda_{n}\right) x_{n}=y_{n} \in \partial \varphi\left(x_{n}\right)$. Consequently

$$
\left[\left(1-\lambda_{n}\right) x_{n}, z_{0}-x_{n}\right] \leq \varphi\left(z_{0}\right)-\varphi\left(x_{n}\right)
$$

By hypothesis, when $\left\|x_{n}\right\| \geq \max \left\{r,\left\|z_{0}\right\|\right\}$, we obtain the following contradiction:

$$
0 \leq\left(\lambda_{n}-1\right)\left(\left\|x_{n}\right\|-\left\|z_{0}\right\|\right)\left\|x_{n}\right\| \leq \varphi\left(z_{0}\right)-\varphi\left(x_{n}\right)<0 .
$$

This contradiction allows us to conclude that there exists $R>0$ such that if $x \in D(\partial \varphi)$ with $\|x\| \geq R$ then

$$
\sup _{y \in \partial \varphi(x)} G(x-y, x) \leq G(x, x)
$$

Since $H$ has the (FPP), from Theorem 3.1 we conclude that $0_{H} \in R(\partial \varphi)$; that is, there exists $x \in H$ such that

$$
0_{H} \in \partial \varphi(x)
$$

and therefore $x$ is an absolute minimum of $\varphi$.

If $X$ has the (FPP), $A: D(A) \rightarrow 2^{X}$ is an accretive operator with the range condition, and $\overline{D(A)}$ is convex and bounded, then, $0_{X} \in R(A)$; see [8]. For the case that $\overline{D(A)}$ is not bounded we have the following result.

Theorem 3.7. Let $X$ be a Banach space. Suppose that $G: X \times X \rightarrow \mathbb{R}$ is a mapping satisfying conditions ( $g 1)-(g 4)$.

If $X$ has the (FPP), $A: D(A) \rightarrow 2^{X}$ is an accretive operator with the range condition, $\overline{D(A)}$ is convex, and $A$ satisfies condition (3.1), then $0_{X} \in R(A)$.

Proof. Since $A$ is accretive with the range condition, then the following two conditions hold:

(i) $\overline{D(A)}=\bigcap_{\curlywedge>0} R(I+\lambda A)$,

(ii) $g:=(I+A)^{-1}: R(I+A) \rightarrow D(A)$ is a nonexpansive mapping. 
Fix $x_{0} \in \overline{D(A)}$. For each positive integer $n$, from (i) there exist $x_{n} \in D(A)$ and $y_{n} \in A\left(x_{n}\right)$ such that

$$
x_{0}=x_{n}+n y_{n}
$$

Hence, $x_{0}=(1+n) x_{n}+n\left(y_{n}-x_{n}\right)$. It follows that

$$
x_{n}-y_{n}=\frac{n+1}{n} x_{n}-\frac{1}{n} x_{0}
$$

We claim that $\left(x_{n}\right)$ is a bounded sequence. Indeed, otherwise we can assume that there exists a subsequence $\left(x_{n_{k}}\right)$ of $\left(x_{n}\right)$ such that $\left\|x_{n_{k}}\right\| \rightarrow \infty$. Without loss of generality we may assume that $\left\|x_{n_{k}}\right\| \geq \max \{R, S, t\}, k \in \mathbb{N}$, where the constants $S$ and $t$ are given in the definitions of conditions (g2) and (g4), respectively.

Therefore, we have

$$
\begin{aligned}
0<G\left(x_{n_{k}}, x_{n_{k}}\right) & =G\left(\frac{n_{k}}{n_{k}+1}\left(x_{n_{k}}-y_{n_{k}}\right)+\frac{1}{n_{k}+1} x_{0}, x_{n_{k}}\right) \\
& \leq \frac{n_{k}}{n_{k}+1} G\left(x_{n_{k}}-y_{n_{k}}, x_{n_{k}}\right)+\frac{1}{n_{k}+1} G\left(x_{0}, x_{n_{k}}\right) .
\end{aligned}
$$

Consequently,

$$
G\left(x_{n_{k}}, x_{n_{k}}\right)<\left(\frac{n_{k}}{n_{k}+1} G\left(x_{n_{k}}, x_{n_{k}}\right)+\frac{1}{n_{k}+1} G\left(x_{n_{k}}, x_{n_{k}}\right)\right)=G\left(x_{n_{k}}, x_{n_{k}}\right) .
$$

This is a contradiction which proves our claim.

Since $\left(x_{n}\right)$ is a bounded sequence, it is clear that $\left(y_{n}\right)$ goes to $0_{X}$ as $n$ goes to infinity.

Now we claim that $g$ has a bounded a.f.p. sequence. Indeed, consider for each positive integer $n, w_{n}=x_{n}+y_{n}$. It is not difficult to see that $g\left(w_{n}\right)=x_{n}$ because $y_{n} \in A\left(x_{n}\right)$. In this case, we obtain

$$
w_{n}-g\left(w_{n}\right)=y_{n} \longrightarrow 0_{X}
$$

Finally, if we call $r_{0}=\lim \sup \left\|w_{n}-x_{0}\right\|$, we obtain that the following set

$$
K=\left\{x \in \overline{D(A)}: \lim \sup \left\|w_{n}-x\right\| \leq r_{0}\right\}
$$

is bounded closed convex and $g$-invariant. Thus, since $X$ enjoys the (FPP), there exists $z \in K$ such that $z=g(z)$ and then $0_{X} \in A(z)$.

Remark 3.8. If we check the proof of Theorem 3.7, we may notice that such theorem still holds if we omit conditions (g3) and (g4) but we add $0_{X} \in \overline{D(A)}$. 
Corollary 3.9. Let $X$ be a Banach space. Suppose that $G: X \times X \rightarrow \mathbb{R}$ is a mapping satisfying conditions ( $g 1)-(g 4)$.

If $X$ has the (FPP), $A: D(A) \rightarrow 2^{X}$ is an accretive operator with the range condition, $\overline{D(A)}$ is convex, and $A$ satisfies condition (3.12), then $0_{X} \in R(A)$.

\section{Fixed Point Results}

Theorem 4.1. Let $X$ be a Banach space with the (FPP). Suppose that $G: X \times X \rightarrow \mathbb{R}$ is a mapping satisfying conditions ( $g 1)$ and ( $g 2$ ). Let $C$ be a closed convex and unbounded subset of $X$ with $0_{X} \in C$. Let $T: C \rightarrow X$ be a continuous pseudocontractive mapping. Assume that the following conditions are satisfied.

(a) $T$ is weakly inward on $C$.

(b) There exists $R>0$ such that for every $x \in C$ with $\|x\| \geq R$ the inequality

$$
G(T(x), x) \leq G(x, x)
$$

holds.

Then $T$ has a fixed point in $C$.

Proof. Since $T: C \rightarrow X$ is a continuous, pseudocontractive mappings weakly inward on $C$, then $A=I-T: C \rightarrow X$ is an accretive operator with the range condition (see $[11,15]$ ).

Let us see that condition (3.1) is satisfied. Indeed, if $x \in C$ with $\|x\| \geq R$,

$$
\sup _{y \in A x} G(x-y, x)=G(x-(x-T(x)), x)=G(T(x), x) .
$$

The above equality along with (4.1) allows us conclude that condition (3.1) holds.

On the other hand, since $0_{X} \in C=\overline{D(A)}$, by Remark 3.8 and following the same argument developed in the proof of Theorem 3.7, it is not difficult to see that

$$
g:=(I+A)^{-1}: R(I+A) \longrightarrow C
$$

has a bounded a.f.p. sequence $\left(w_{n}\right)$, and thus, if we call $r_{0}=\lim \sup \left\|w_{n}\right\|$, we obtain that the set

$$
K=\left\{x \in C: \lim \sup \left\|w_{n}-x\right\| \leq r_{0}\right\}
$$

is bounded closed convex and $g$-invariant. Thus, since $X$ enjoys the (FPP), there exists $z \in K$ such that $z=g(z)$ and then $0_{X}=A(z)=z-T(z)$. 
Corollary 4.2. Let $X$ be a Banach space with the (FPP). Suppose that $G: X \times X \rightarrow \mathbb{R}$ is a mapping satisfying conditions ( $g 1)$ and ( $g 2$ ). Let $C$ be a closed convex and unbounded subset of $X$ with $0_{X} \in C$. If $T: C \rightarrow X$ is a continuous pseudocontractive mapping weakly inward on $C$ and

$$
\lim _{\substack{x \in C,\|x\| \rightarrow \infty}} \frac{G(T(x), x)}{G(x, x)}<1,
$$

then $T$ has a fixed point in $C$.

Proof. Clearly inequality (4.5) implies condition (4.1).

Corollary 4.3. Let $X$ be a Banach space with the (FPP). Suppose that $G: X \times X \rightarrow \mathbb{R}$ is a mapping satisfying conditions (g1)-(g4). Let $C$ be a closed convex and unbounded subset of $X$. If $T: C \rightarrow X$ is a continuous pseudocontractive mapping weakly inward on $C$ and satisfies condition (4.1), then $T$ has a fixed point in $C$.

Proof. From the above theorem, we know that $A=I-T: C \rightarrow X$ is an accretive operator with the range condition and with condition (3.1). Therefore by Theorem 3.7 we obtain the result.

Remark 4.4. In order to give an alternative proof of Corollary 4.3, it is enough to see that condition (4.5) implies that $T$ has an a.f.p. sequence $\left(x_{n}\right)$, and thus, using [16, Theorem 4.3], we obtain the same conclusion. In this case, if we assume that $X$ is a reflexive Banach space and $I-T$ is demiclosed at zero, then we can remove the assumption on the (FPP) for the space $X$. Nevertheless, it is well known that there exist nonreflexive Banach spaces with the FPP (see [13]). On the other hand, if $X$ is a reflexive Banach space such that for every nonexpansive mapping, say $T$, the mapping $I-T$ is demiclosed at $0_{X}$, then the Banach space has the FPP.

Remark 4.5 (Theorem 3.2 in [6] reads). Let $X$ be a reflexive Banach space. Suppose that $G: X \times$ $X \rightarrow \mathbb{R}$ satisfies conditions (G1), (G2), (G3), and (G4). Let $C \subseteq X$ be a nonempty unbounded closed convex set. If $T: C \rightarrow X$ is a nonexpansive mapping such that $T(C) \subset C, I-T$ is demiclosed and

$$
\lim _{\substack{x \in C,\|x\| \rightarrow \infty}} \frac{G\left(T(x)-x_{0}, x\right)}{\|x\|^{2}}<1
$$

for some $x_{0} \in C$, then $T$ has a fixed point in $C$.

Notice that Corollary 4.3 generalizes this theorem in several senses.

(i) Our assumptions (g1)-(g4) on mapping $G$ are weaker than the corresponding in that theorem.

(ii) Every nonexpansive mapping is in fact continuous and pseudocontractive.

(iii) The inwardness condition is more general than the assumption $T(C) \subset C$. 
Fixed Point Theory and Applications

(iv) Condition (4.6) implies that

$$
\begin{aligned}
\frac{G(T(x), x)}{\|x\|^{2}} & \leq \frac{G\left(T(x)-x_{0}+x_{0}, x\right)}{\|x\|^{2}} \\
& \leq \frac{G\left(T(x)-x_{0}, x\right)+G\left(x_{0}, x\right)}{\|x\|^{2}} \\
& \leq \frac{G\left(T(x)-x_{0}, x\right)}{\|x\|^{2}}+\frac{M\left\|x_{0}\right\|\|x\|}{\|x\|^{2}} .
\end{aligned}
$$

Interchanging the roles of $x_{0}$ and $0_{\mathrm{X}}$ we can conclude that

$$
\lim _{\substack{x \in C_{1} \\\|x\| \rightarrow \infty}} \frac{G(T(x), x)}{\|x\|^{2}}=\lim _{\substack{x \in C_{1} \\\|x\| \rightarrow \infty}} \frac{G\left(T(x)-x_{0}, x\right)}{\|x\|^{2}}
$$

for every $x_{0} \in X$. Therefore, there exists $R>0$ such that if $\|x\| \geq R$, then

$$
G(T(x), x) \leq\|x\|^{2} \leq G(x, x),
$$

which is just condition (4.1) of Theorem 4.1.

In the same sense, Theorem 4.1 is a generalization of Theorem 3.1 of [6].

Corollary 4.6. Let $X$ be a Banach space with the (FPP). Let $C$ be a closed convex and unbounded subset of $X$ such that $0_{X} \in C$. Let $T: C \rightarrow X$ be a continuous pseudocontractive mapping. Assume that the following conditions are satisfied.

(a) $T$ is weakly inward on $C$.

(b) There exists $R>0$ such that for every $x \in C \backslash B_{R}$ and for every $\lambda>1, T(x) \neq \lambda x$.

Then $T$ has a fixed point in $C$.

Proof. It is enough to apply Theorem 4.1, where $G: X \times X \rightarrow \mathbb{R}$ is defined by

$$
G(x, y)= \begin{cases}\lambda, & \text { if } x=\lambda y, \lambda>0, x \neq 0, \\ 0, & \text { otherwise, }\end{cases}
$$

and if $x \in C \backslash B_{R}$, then $G(T x, x) \leq 1=G(x, x)$.

Remark 4.7. Notice that the above condition (b) is similar to the well-known Leray-Schauder boundary condition. Some results of this type can be found in [17-19].

Corollary 4.8. Let $X$ be a Banach space with the (FPP). Let $C$ be a closed convex and unbounded subset of $X$. If $T: C \rightarrow X$ is a continuous pseudocontractive mapping weakly inward on $C$ and for every $x \in C$ and $\|x\|$ large enough

$$
\left\|T(x)-x_{0}\right\| \leq\left\|x-x_{0}\right\|
$$

for some $x_{0} \in X$, then $T$ has a fixed point in $C$. 
Proof. Let $G: X \times X \rightarrow \mathbb{R}$ be the function defined by $G(x, y)=\left\langle x, y-x_{0}\right\rangle_{+}$. It is clear that $G$ satisfies conditions (g1) and (g3) . Moreover,

$$
G(x, x)=\left\langle x, x-x_{0}\right\rangle_{+}=j(x)
$$

for some $j \in J\left(x-x_{0}\right)$. Therefore,

$$
G(x, x)=\left\langle x, x-x_{0}\right\rangle_{+}=j(x)=j\left(x-x_{0}\right)+j\left(x_{0}\right) \geq\left\|x-x_{0}\right\|^{2}-\left\|x-x_{0}\right\|\left\|x_{0}\right\| .
$$

Since $x_{0}$ is a fix element of $X$, clearly there exists $S>0$ such that $G(x, x)>0$ whenever $\|x\| \geq S$. This means that $G$ satisfies (g2).

To see that $G$ satisfies condition (g4) we argue as follows.

Given a fix $y \in X$, we know that $|G(y, x)| \leq\|y\|\left\|x-x_{0}\right\|$.

Since $\left\|x-x_{0}\right\|-\left\|x_{0}\right\| \rightarrow \infty$ as $\|x\| \rightarrow \infty$, we can find $t>0$ such that $\left(\left\|x-x_{0}\right\|-\left\|x_{0}\right\|\right)>$ $\|y\|$ for every $\|x\| \geq t$.

Then,

$$
|G(y, x)| \leq\|y\|\left\|x-x_{0}\right\|<\left(\left\|x-x_{0}\right\|-\left\|x_{0}\right\|\right)\left\|x-x_{0}\right\| \leq G(x, x)
$$

Now, we will see that $G$ satisfies inequality (4.1) in Corollary 4.3. Indeed, if $\|x\| \geq R$, we have, for some $j \in J\left(x-x_{0}\right)$, that

$$
\begin{aligned}
G(T x, x) & =\left\langle T x, x-x_{0}\right\rangle_{+}=j(T x)=j\left(T x-x_{0}\right)+j\left(x_{0}\right) \\
& \leq\left\|T x-x_{0}\right\|\left\|x-x_{0}\right\|+j\left(x_{0}\right) \\
& \leq\left\|x-x_{0}\right\|^{2}+j\left(x_{0}\right)=j(x) \\
& \leq\left\langle x, x-x_{0}\right\rangle_{+} \\
& =G(x, x) .
\end{aligned}
$$

Thus the conclusion follows from Corollary 4.3.

Remark 4.9. In the case that for all $x \in C,\left\|T(x)-x_{0}\right\| \leq\left\|x-x_{0}\right\|$, then the mapping $T$ is said to have $x_{0}$ as a center; see [20], where some fixed point theorems are given for this class of mappings.

On the other hand, in [21, Corollary 1.6, page 54] one can read a similar condition, where the domain of the mapping is required to be bounded. see that

If $T: C \rightarrow X$ is asymptotically contractive in the sense due to Penot, then it is easy to

$$
\limsup _{x \in C,\|x\| \rightarrow \infty} \frac{\|T(x)\|}{\|x\|}<1
$$

which implies condition (4.11) of Corollary 4.8 for $x_{0}=0_{X}$, and therefore Penot's fixed point theorem is a consequence of Corollary 4.8 . 
Example 4.10. Next, we are concerned with the solvability of the following Hammerstein's integral equation:

$$
u(t)=w(t)+\int_{\Omega} \zeta(t, s) f(s, u(s)) d s
$$

in $L^{p}(\Omega)$. Here $1<p<\infty, \Omega$ is a bounded domain of $\mathbb{R}^{n}$, such that its Lebesgue's measure $\mu(\Omega)=1$, and $w \in L^{p}(\Omega)$. Suppose that $\zeta$ and $f$ satisfy the following conditions:

(1) $f: \Omega \times \mathbb{R} \rightarrow \mathbb{R}$ is a Carathéodory function,

(2) $|f(s, x)| \leq a(s)+b|x|$, where $a \in L^{p}(\Omega)$ and $b \geq 0$,

(3) $|f(s, x)-f(s, y)| \leq k|x-y|$,

(4) the function $\zeta: \Omega \times \Omega \rightarrow \mathbb{R}$ is strongly measurable and $\int_{\Omega} \zeta(\cdot, s) u(s) d s \in L^{p}(\Omega)$ whenever $u \in L^{p}(\Omega)$,

(5) there exists a function $\tau: \Omega \rightarrow \mathbb{R}$, belonging to $L^{p}(\Omega)$ such that $|\zeta(t, s)| \leq \tau(t)$ for all $(t, s) \in \Omega \times \Omega$,

(6) $k\|\tau\|_{p} \leq 1$ and $b\|\tau\|_{p}<1$.

Proposition 4.11. Assume that conditions (1)-(6) are satisfied, then problem (4.17) has at least one solution in $L^{p}(\Omega)$.

Proof. First notice that (4.17) may be written in the form $u=T(u)$ where $T$ is given by

$$
T: L^{p}(\Omega) \longrightarrow L^{p}(\Omega): u \longrightarrow T(u)(t):=w(t)+\int_{\Omega} \zeta(t, s) f(s, u(s)) d s .
$$

Let us see that $T$ satisfies the conditions of Corollary 4.8. In this sense, we are going to prove that $T$ is a nonexpansive mapping. Indeed,

$$
\begin{aligned}
\|T(u)-T(v)\|_{p}^{p} & =\int_{\Omega}\left|\int_{\Omega} \zeta(t, s)(f(s, u(s))-f(s, v(s))) d s\right|^{p} d t \\
& \leq \int_{\Omega}\left(\int_{\Omega}|\zeta(t, s)(f(s, u(s))-f(s, v(s)))| d s\right)^{p} d t \\
& \leq \int_{\Omega}|\tau(t)|^{p}\left(\int_{\Omega}|f(s, u(s))-f(s, v(s))| d s\right)^{p} d t \\
& \leq \int_{\Omega}|\tau(t)|^{p}\left(\int_{\Omega} k|u(s)-v(s)| d s\right)^{p} d t .
\end{aligned}
$$

Since $\mu(\Omega)=1$, by Holder's inequality, we obtain that

$$
\|T(u)-T(v)\|_{p} \leq k\|\tau\|_{p}\|u-v\|_{p} \leq\|u-v\|_{p} .
$$


Finally, we are going to show that there exists $R>0$ such that if $\|u\|_{p} \geq R$, then $\|T(u)\|_{p} \leq\|u\|_{p}$. Indeed, we know that

$$
\|T(u)\|_{p} \leq\|w\|_{p}+\left\|\int_{\Omega} \zeta(\cdot, s) f(s, u(s)) d s\right\|_{p}^{\prime}
$$

hence,

$$
\begin{aligned}
\left\|\int_{\Omega} \zeta(\cdot, s) f(s, u(s)) d s\right\|_{p}^{p} & =\int_{\Omega}\left|\int_{\Omega} \zeta(t, s) f(s, u(s)) d s\right|^{p} d t \\
& \leq \int_{\Omega}\left(\int_{\Omega}|\tau(t)|(a(s)+b|u(s)|) d s\right)^{p} d t .
\end{aligned}
$$

Applying again Holder's inequality, we derive that

$$
\left\|\int_{\Omega} \zeta(\cdot, s) f(s, u(s)) d s\right\|_{p} \leq\left(\|a\|_{p}+b\|u\|_{p}\right)\|\tau\|_{p} .
$$

Moreover, it is clear that

$$
\lim _{\|u\|_{p} \rightarrow \infty} \frac{\|w\|_{p}+\left(\|a\|_{p}+b\|u\|_{p}\right)\|\tau\|_{p}}{\|u\|_{p}}=b\|\tau\|_{p}<1
$$

therefore there exists $R>0$ such that if $\|u\|_{p} \geq R$, then $\|T(u)\|_{p} \leq\|u\|_{p}$ as we claimed.

Notice that if $k\|\tau\|_{p}=1$ then, Corollary 3 in [4] does not apply because under this condition we cannot guarantee that $T$ is asymptotically contractive on $L^{p}(\Omega)$.

Let $C$ be a closed convex subset of a Banach space $X$. A family of mappings $\{T(t)$ : $C \rightarrow C: t \geq 0\}$ is called a one-parametric strongly continuous semigroup of nonexpansive mappings (nonexpansive semigroup, for short) on $C$ if the following assumptions are satisfied:

(1) $T(s+t)=T(s) \circ T(t)$ for all $s, t \geq 0$,

(2) for each $x \in C$, the mapping $t \mapsto T(t) x$ from [0, $\infty$ [ into $C$ is continuous,

(3) for each $t \geq 0, T(t): C \rightarrow C$ is a nonexpansive mapping.

In the next result we study when a nonexpansive semigroup has a common fixed point.

Theorem 4.12. Let $X$ be a Banach space with the (FPP). Suppose that $G: X \times X \rightarrow \mathbb{R}$ is a mapping satisfying conditions (g1)-(g4). Let $C$ be a closed convex and unbounded subset of $X$. If $\{T(t): C \rightarrow$ $C: t \geq 0\}$ is a nonexpansive semigroup such that there exist $\alpha, \beta \in] 0, \infty[$ with $(\alpha / \beta) \in \mathbb{R} \backslash \mathbb{Q}$ satisfying that

$$
\max \{G(T(\alpha) x, x) ; G(T(\beta) x, x)\} \leq G(x, x)
$$

whenever $x \in C$ large enough, then the semigroup has a least one common fixed point. 
Proof. By Theorem 1 of [22] in order to get the conclusion it is enough to show that, given $\lambda \in(0,1)$, the mapping $T_{\lambda}: C \rightarrow C$ defined by

$$
T_{\lambda}(x)=\lambda T(\alpha)(x)+(1-\lambda) T(\beta)(x)
$$

has a fixed point.

By hypotheses we know that there exists $R>0$ such that for every $x \in C$ with $\|x\| \geq R$ the inequality

$$
\max \{G(T(\alpha) x, x) ; G(T(\beta) x, x)\} \leq G(x, x)
$$

holds. Since G satisfies conditions (g1)-(g4), we have

$$
\begin{aligned}
G\left(T_{\lambda}(x), x\right) & =G(\lambda T(\alpha)(x)+(1-\lambda) T(\beta)(x), x) \\
& \leq G(\lambda T(\alpha)(x), x)+G((1-\lambda) T(\beta)(x), x) \\
& \leq \lambda G(T(\alpha)(x), x)+(1-\lambda) G(T(\beta)(x), x) \\
& \leq G(x, x) .
\end{aligned}
$$

The above inequality means that $T_{\mathcal{\lambda}}$ satisfies the conditions of Corollary 4.3 and therefore $T_{\lambda}$ has a fixed point, which implies by Theorem 1 of [22] that the semigroup has a common fixed point.

Corollary 4.13. Let $X$ be a Banach space with the (FPP). Let $C$ be a closed convex and unbounded subset of $X$. If $\{T(t): C \rightarrow C: t \geq 0\}$ is a nonexpansive semigroup such that there exist $x_{0} \in X$, $\alpha, \beta \in] 0, \infty[$ with $\alpha / \beta \in \mathbb{R} \backslash \mathbb{Q}$ satisfying that

$$
\max \left\{\left\|T(\alpha) x-x_{0}\right\| ;\left\|T(\beta) x-x_{0}\right\|\right\} \leq\left\|x-x_{0}\right\|
$$

whenever $x \in C$ large enough, then the semigroup has a least one common fixed point.

Proof. It is enough to apply the above theorem with $G(x, y)=\left\langle x, y-x_{0}\right\rangle_{+}$(see the proof of Corollary 4.8).

We conclude this section by presenting a corollary of Theorem 4.1 which guarantees the existence of positive eigenvalues.

Corollary 4.14. Let $X$ be a Banach space with the (FPP). Suppose that $G: X \times X \rightarrow \mathbb{R}$ is a mapping satisfying conditions ( $g 1)$ and ( $g 2$ ). Let $C$ be a closed convex and unbounded subset of $X$ with $0_{X} \in C$. Let $T: C \rightarrow C$ be a continuous pseudocontractive mapping. Assume that the following conditions are satisfied.

(a) $T\left(0_{X}\right) \neq 0_{X}$.

(b) There exists $R>0$ such that for every $x \in C$ with $\|x\| \geq R$ the inequality

$$
G(T(x), x) \leq l G(x, x)
$$

holds for some $l \geq 0$.

Then any $\lambda \geq \max \{l, 1\}$ is an eigenvalue of $T$ associated to an eigenvector in $C$. 
Proof. Consider a fixed $\lambda \geq \max \{l, 1\}$. Let us see that $(1 / \lambda) T$ is a continuous pseudocontractive mapping such that $(1 / \lambda) T(C) \subseteq C$. Indeed, since $0_{X} \in C, C$ is convex, $\lambda \geq 1$, and $T(C) \subseteq C$, then $(1 / \lambda) T(C) \subseteq C$.

To see that $(1 / \lambda) T$ is a pseudocontractive mapping, it is enough to prove that $I-(1 / \lambda) T$ is an accretive mapping:

$$
\begin{aligned}
\langle x- & \left.\frac{1}{\lambda} T(x)-\left(y-\frac{1}{\lambda} T(y)\right), x-y\right\rangle_{+} \\
& =\left\langle\frac{1}{\lambda}(x-T(x)-(y-T(y)))+\left(1-\frac{1}{\lambda}\right)(x-y), x-y\right\rangle_{+} \\
& \geq \frac{1}{\lambda}\langle x-T(x)-(y-T(y)), x-y\rangle_{+}+\left(1-\frac{1}{\lambda}\right)\|x-y\|^{2} \geq 0 .
\end{aligned}
$$

The above inequality holds since $T$ is a pseudocontractive mapping and therefore $\langle x-T(x)-(y-T(y)), x-y\rangle_{+} \geq 0$.

Finally, if $x \in C$ with $\|x\| \geq R$, we have

$$
G\left(\frac{1}{\lambda} T(x), x\right) \leq \frac{1}{\lambda} G(T x, x) \leq \frac{l}{\lambda} G(x, x) \leq G(x, x) .
$$

The above facts show that $(1 / \lambda) T$ is under the assumption of Theorem 4.1 and hence there exists $x_{\curlywedge} \in C \backslash\{0\}$ such that $T\left(x_{\curlywedge}\right)=\lambda x_{\lambda}$.

\section{Acknowledgments}

Both authors were partially supported by MTM 2009-10696-C02-02. This work is dedicated to Professor W. A. Kirk.

\section{References}

[1] W. A. Kirk, "A fixed point theorem for mappings which do not increase distances," The American Mathematical Monthly, vol. 72, pp. 1004-1006, 1965.

[2] W. A. Kirk and B. Sims, Eds., Handbook of Metric Fixed Point Theory, Kluwer Academic Publishers, Dordrecht, The Netherlands, 2001.

[3] K. Goebel and W. A. Kirk, Topics in Metric Fixed Point Theory, vol. 28 of Cambridge Studies in Advanced Mathematics, Cambridge University Press, Cambridge, UK, 1990.

[4] J.-P. Penot, "A fixed-point theorem for asymptotically contractive mappings," Proceedings of the American Mathematical Society, vol. 131, no. 8, pp. 2371-2377, 2003.

[5] M. Altman, "A fixed point theorem in Hilbert space," Bulletin de l'Academie Polonaise des Sciences, vol. 5, pp. 19-22, 1957.

[6] G. Isac and S. Z. Németh, "Fixed points and positive eigenvalues for nonlinear operators," Journal of Mathematical Analysis and Applications, vol. 314, no. 2, pp. 500-512, 2006.

[7] W. A. Kirk and R. Schöneberg, "Zeros of $m$-accretive operators in Banach spaces," Israel Journal of Mathematics, vol. 35, no. 1-2, pp. 1-8, 1980.

[8] J. García-Falset and S. Reich, "Zeroes of accretive operators and the asymptotic behavior of nonlinear semigroups," Houston Journal of Mathematics, vol. 32, no. 4, pp. 1197-1225, 2006.

[9] C. Morales, "Nonlinear equations involving $m$-accretive operators," Journal of Mathematical Analysis and Applications, vol. 97, no. 2, pp. 329-336, 1983. 
[10] I. Cioranescu, Geometry of Banach Spaces, Duality Mappings and Nonlinear Problems, vol. 62 of Mathematics and Its Applications, Kluwer Academic Publishers, Dordrecht, The Netherlands, 1990.

[11] R. H. Martin Jr., "Differential equations on closed subsets of a Banach space," Transactions of the American Mathematical Society, vol. 179, pp. 399-414, 1973.

[12] J. R. Giles, "Classes of semi-inner-product spaces," Transactions of the American Mathematical Society, vol. 129, pp. 436-446, 1967.

[13] G. Lumer, "Semi-inner-product spaces," Transactions of the American Mathematical Society, vol. 100, pp. 29-43, 1961.

[14] H. Brézis, Opérateurs maximaux monotones et semi-groupes de contractions dans les espaces de Hilbert, North-Holland Mathematics Studies, no. 5. Notas de Matemática (50), North-Holland, Amsterdam, The Netherlands, 1973.

[15] F. E. Browder, "Nonlinear mappings of nonexpansive and accretive type in Banach spaces," Bulletin of the American Mathematical Society, vol. 73, pp. 875-882, 1967.

[16] J. García-Falset, "Fixed points for mappings with the range type condition," Houston Journal of Mathematics, vol. 28, no. 1, pp. 143-158, 2002.

[17] C. González, A. Jiménez-Melado, and E. Llorens-Fuster, "A Mönch type fixed point theorem under the interior condition," Journal of Mathematical Analysis and Applications, vol. 352, no. 2, pp. 816-821, 2009.

[18] W. A. Kirk, "Fixed point theorems for nonexpansive mappings satisfying certain boundary conditions," Proceedings of the American Mathematical Society, vol. 50, pp. 143-149, 1975.

[19] C. H. Morales, "The Leray-Schauder condition for continuous pseudo-contractive mappings," Proceedings of the American Mathematical Society, vol. 137, no. 3, pp. 1013-1020, 2009.

[20] J. García-Falset, E. Llorens-Fuster, and S. Prus, "The fixed point property for mappings admitting a center," Nonlinear Analysis: Theory, Methods \& Applications, vol. 66, no. 6, pp. 1257-1274, 2007.

[21] A. Granas and J. Dugundji, Fixed Point Theory, Springer Monographs in Mathematics, Springer, New York, NY, USA, 2003.

[22] T. Suzuki, "Common fixed points of one-parameter nonexpansive semigroups," The Bulletin of the London Mathematical Society, vol. 38, no. 6, pp. 1009-1018, 2006. 